\title{
DEVELOPMENT AND TESTING OF A SIMULATION GAME ON WASTE ELIMINATION USING LEAN PRACTICES
}

\author{
Shaurya Bhatnagar ${ }^{1}$ and Ganesh Devkar ${ }^{2}$
}

\begin{abstract}
Lean concepts of waste elimination and analysis of Value-Adding (VA) and Non-ValueAdding (NVA) activities holds the potential for improved processes in a construction project and enhanced value creation for the client. Simulation games can be effectively used to impart knowledge about these concepts and tap the potential of lean philosophy in the construction industry. This paper reports the development and testing of a simulation game that focuses on waste elimination and value maximisation using lean principles. This paper chronicles the details of setting game requirements, prototype design, material selection, sequence of work, room set up, roles and scenarios and rules for different rounds. The simulation game consisted of three rounds. Round 1 involved traditional construction processes in which, lean wastes are evident, which adversely affects variables like time, cost and quality. In Rounds 2 and 3, various lean practices are introduced, with an aim to eliminate waste and to understand value-adding and non-valueadding activities. The developed simulation was tested with post graduate students at CEPT University, India. The post simulation discussion indicated that the simulation game resulted in enhanced understanding on waste, value and lean practices. This simulation game can be further enhanced by integrating aspects of value stream mapping of construction process.
\end{abstract}

\section{KEYWORDS}

Lean, simulation game, waste elimination, value maximisation, cost, lead time.

\section{INTRODUCTION}

The construction industry has been undergoing significant transformations in the areas of contracting, design management, and facility management globally to deal with challenges such as design defects, schedule delays, cost overruns, complex workflows, unreliability in output, coordination issues, inventory mismatch problems, and wastage of materials. In this context, lean philosophy has been gaining immense attention among the stakeholders of the construction industry and it is expected to play a key role in this transformation process.

Lean philosophy includes a set of tools, principles, and production techniques that identify and eliminate waste through continual improvements in processes. This lean

1 Post Graduate Student, Faculty of Technology, CEPT University, Ahmedabad - 380009, India +919871929730, shauryabhatnagar2012@gmail.com, orcid.org/0000-0003-3296-478X

2 Associate Professor, Faculty of Technlogy, CEPT University, Ahmedabad - 380009, India +919099010303, ganesh.devkar@ cept.ac.in, orcid.org/0000-0002-5482-1221 
philosophy focuses on four critical concepts - waste, value, continuous improvement, and respect (Rybkowski et al., 2018). Waste is the pivotal concept and has been defined by Toyota as "anything different from the absolute minimum amount of resources of materials, equipment, and manpower necessary to add value to the product" (Alarcón, 1995, p. 1). The pioneering work by Koskela (2000) on the adoption of lean philosophy in the construction sector put forth the Transformation-Flow-Value (TFV theory). The TFV theory of production emphasizes on elimination of waste and non-value adding (NVA) activities for better flow management through continuous improvement.

While lean concepts such as flow, value, waste, and value maximisation are promising and can potentially improve the construction processes, a survey of literature shows that the "lack of understanding knowledge on Lean and Complexity of Lean philosophy and terms" continues to be a potential barrier in the adoption of lean principles in the construction sector (Demirkesen et al., 2019, pp. 7-8). The gap between the concept and application of lean philosophy can be bridged by a new way of "Learning by Doing" i.e. Simulation Games. In the construction domain, lean simulation games are considered to be an effective mechanism to impart knowledge on various lean concepts in a clear, realistic, and simplified manner (Hamzeh et al., 2017; Rybkowski et al., 2018).

Bhatnagar (2020) compiled forty-seven lean simulation games from a matrix of Lean Construction Institute (2021) and papers from International Group for Lean Construction (2021) and American Society of Civil Engineers (2021). These games were analysed in terms of their learning outcomes and lean principles. The study showed that important themes such as waste elimination and value maximisation continue to be unexplored and these are not key focus areas of existing lean simulation games. The games such as LEAPCON, House of cards, Dot Simulation, Airplane Game (and its variants) deal with waste along with various other learning objectives but do not hold reduction of waste and analysis of VA/NVA as the key learning outcomes (Pollesch \& Rovinsky, 2017; Rybkowski et al., 2018).

This paper analyses the development and testing of a simulation game to impart knowledge on waste elimination and value maximisation using various lean practices in the construction domain. This game is inspired from Airplane game developed by Visionary Products USA, Inc. (2021) which teaches teamwork, pull production, and the impact of supply chain logistics.

\section{GAME DEVELOPMENT}

The main intention of the proposed game is to familarise participants with waste elimination and value maximisation concepts with the help of various lean practices. Each team has to complete the target of constructing eight Lego ${ }^{\mathrm{TM}}$ houses within the stipulated time of eight minutes to get cash points for houses that are defect free. The game is played in three rounds with each round showing continuous improvement in the workflow process due to reduction in wastes by use of 5S, Supermarket, Kanban, Heijunka box, and pull planning. At the end of 8 minutes in each round, the numbers of houses constructed by each team are counted and each house is inspected. The team with highest cashpoints is declared a winner. The three keypoints - doing more with less effort, acting smarter rather than harder and reducing lean wastes are used to reach the target.

\section{TEAM COMPOSITION}

The game includes the entire cycle of a typical house construction. Each team comprises 8 players - one contractor head, one safety officer, one quality manager, and one person 
each for different trades such as foundation laying, column casting, blockwork, roof work and service laying. Additionally, 1 assistant and 2 timekeepers per team are required. The game can be played among 2 - 4 teams with one instructor. At the start of each round, the assistant conveys information about the number of targeted houses to be constructed and time limit for each round and distributes materials and templates to the Contractor Head of each team. The task of house construction is then performed by each team in accordance with the instructions. The timekeepers note the time in the format card for each team and display the results in the form of a Gantt chart on a spreadsheet on a computer. The instructor conducts debriefing sessions for players at the end of each round with the help of the assistant and timekeepers.

\section{Prototype Design}

The prototype "house" is chosen for the game. The design is inspired from the House of Lean model and comprises foundation, columns, block walls, roofing and services (Liker, 2004). The design units are Lego ${ }^{\mathrm{TM}}$ blocks of required shapes, sizes, colours according to different design codes. While designing the prototype, the number of blocks used by each trade are kept different, creating an imbalance in their workload. There are four design codes - D1, D2, D3, D4. Each design code has the same constructibility of prototype but the colours of all blocks differ for each code. All the blocks are green for D1, red for D2, blue for D3 and yellow for D4. This is done to induce interest and concentration in the game. All teams are required to construct the houses with D1, D2, D3, D4 and the sequence of using design code will be based on a special symbol given to each team. Each sequence is denoted by the four special symbols - spade, club, diamond and heart - as shown below.

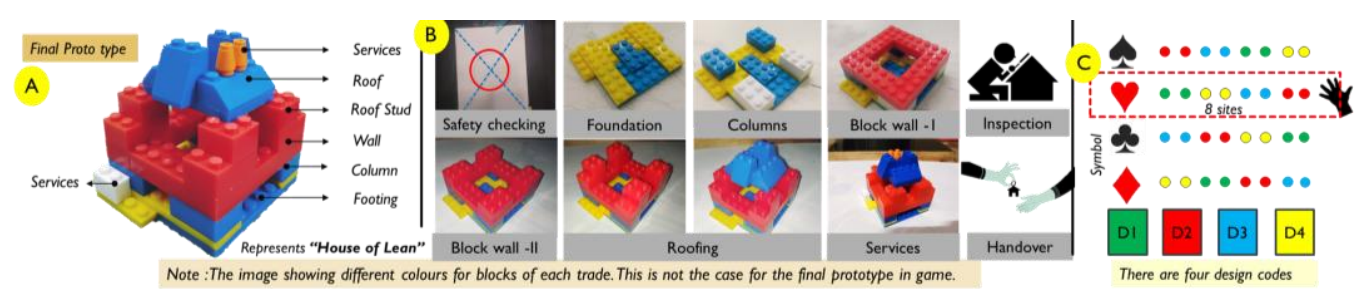

Figure 1: A - Prototype Design, B - Sequence of work, C - Sequence of design codes

\section{MATERIALS REQUIRED}

Each team is provided 1 standard 52-card deck, 5 small bowls, $200 \mathrm{~g}$ of shredded paper, 3 small packets of colourful small beads, 3 packs of colourful sticky notes of size $7.5 \mathrm{~cm}$ x $7.5 \mathrm{~cm}$ and 1 pack of A4 colourful sheets. Additionally, each team is provided 1 circular paper stencil of $50 \mathrm{~mm}$ radius, 1 cardboard stencil of A4 size with $50 \mathrm{~mm}$ radius circular cut-out in centre, 3-4 sketch pens/markers, 1 steel ruler, 1 adhesive tape with dispenser, 2 stopwatches, 1 compass, 1 scissors and 4 Heijunka boxes. Three copies of various templates such as specification card, format card, costing template and design sheet are provided to all teams. The design sheet has information on prototype design, design code details and sequence of design code to be followed while specification card defines important instructions such as number of targeted houses and time limit for each round to be followed while playing. Format card is a template to note the time readings for each player's entry and exit while playing. Costing template records the cashpoints paid to each trade and profit earned by the team. Pre-formulated excel template and debriefing questionnaire are also provided. Sticky notes are used as cashpoints in all rounds and as Kanban cards in Round 3. The two varieties of blocks are placed in material bowls for 
house construction. One is an original block from the Lego ${ }^{\mathrm{TM}}$ company while the other is sourced locally from a local brand called Peco. As per the specification sheet, blocks with Lego trademark are approved for house construction. The details of Lego and Peco blocks are mentioned in the design sheet. Few blocks for roof work are replaced intentionally for each team with duplicate Peco blocks before the start of the game. Also, some pieces are marked with a marker intentionally as shown in Figure 2, making it a defective piece and these blocks are also mixed with others by assistants. This induces defect/rework waste as the specification card gives strict instructions that the cashpoints shall only be paid for defect-free sites made up of blocks having trademark of Lego and free from any marks.

A

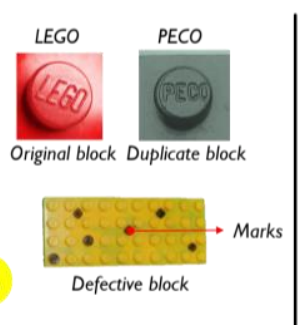

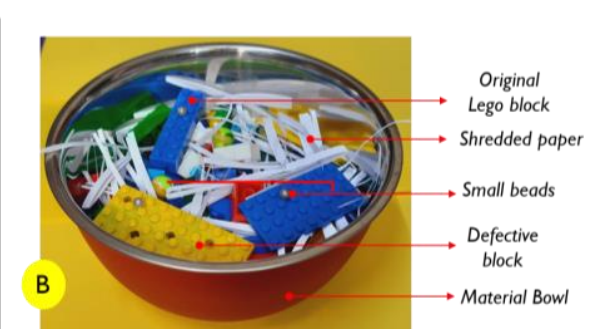

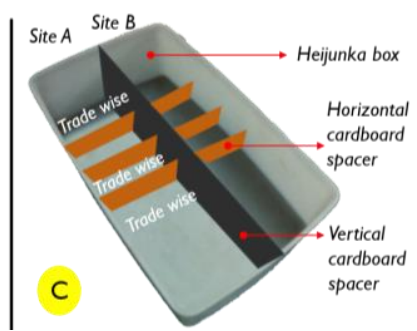

Figure 2: A -Types of blocks, B - Material bowl, C - Heijunka Box

The material bowls are not more than $150 \mathrm{~mm}$ in diameter and depth varies from 50-75 $\mathrm{mm}$. Each bowl contains the exact number of Lego blocks required by the design sheet. Additional materials such as extra Lego blocks of different shapes, sizes, and colours, small beads and shredded paper are also added to the bowl. The bowl design and material mix are intended to introduce difficulty resulting in time and effort wastage in choosing the right Lego blocks. Heijunka boxes are used in Round 2 and 3 intead of material bowls. It is a simple rectangular box with an open top and has vertical and horizontal dividers made up of cardboard spacers. The horizontal rows define the space for each trade while the vertical columns define site-wise division. They are used for $5 \mathrm{~S}$ and resource sharing purposes. The Heijunka box enables easy and fast check and choice of Lego blocks. The time wasted on extra, unnecessary movement can thus be considerably reduced.

\section{SETTING Up OF RoOM}

The game is required to be played in a classroom environment with accessibility to a projector screen and laptop. Each team is given two tables. The smaller table is used as a material station to keep Lego blocks, sheets, bowls, etc. On the right, a minimum of 4 tables (say $1200 * 600 \mathrm{~mm}$ each) are connected together to form a working station. A distance of 1800-2400 $\mathrm{mm}$ is kept between the two stations purposely in Round 1 and subsequently removed in later rounds. The upper half of the working station is left for material movement while the lower half is reserved for the house construction and is the main access to the working station for players. Eight sites (A-H) are marked on the working station with inter-site distance of $600 \mathrm{~mm}$. The movement of players is required to be from left to right while playing. The waiting or queuing space is kept in front of the material station to provide hassle-free entry into working station. Some space for movement is reserved on the upper side of the working station for the quality manager. Seven sticky notes are pasted on the table as money slips to the right of each site. Each trade is given sticky notes of a specific colour. The slips are pasted at the bottom for the last trade and at the top for the first trade. The typical room layout arrangement is shown in Figure 3. 


\section{InSTRUCTIONS To Play}

The simulation starts with the participants dividing themselves into teams of 8 players each and the rest take up the roles of assistant and timekeepers. The tutor assumes the role of the instructor. Each team member is asked to select one of the roles mentioned in the section on team composition. Then, the facilitators give the copies of the specification card, format card, costing template and design sheet along with material to the contractor head of each team. The contractor head chooses one card from the pack of 52 cards. The chosen symbol defines the sequence of the four design codes (D1-D4) to be followed on each site while playing. Each contractor head gets 3000 cash points as advance to maximise profit and minimise expenditure. Any material, once bought, cannot be returned or refunded. The timer starts with the safety officer entering each site to paste A4 sheet and drawing a circle at the centre for the next trades to work. The respective trades join the required Lego blocks as per the design sheet and specification card. The quality manager arrives at the end for inspection, after which, cashpoints are paid to each team for defect-free houses. Timekeepers record the entry and exit of each player. After 8 minutes, the players and timers are paused for recording work-in-progress and completed sites as given in the format card. After the break, the process is continued until eight houses are built and the total time is noted. The costing template is filled by contractor head to determine the profit earned by each team at the end of the rounds. After each round, the instructor conducts debriefing sessions to summarise learnings and experiences. The durations of the three rounds (including debriefing) are approximately 50 minutes, 40 minutes and 35 minutes respectively followed by a small break after each round.

\section{Round 1}

Round 1 represents the traditional way of working depicting several lean wastes. All the material is bought at the initial stage leading to inventory and housekeeping issues. After reaching the work station, each player moves towards the material station for collecting the material and then back to the working station which is $1800-2400 \mathrm{~mm}$ away. Here, time is lost due to wasted movement. A batch size of 4 houses is taken for construction, which means that next trade can only start once 4 sites are completed by the previous trade. Wastage due to waiting and overproduction is experienced by the players. The safety officer pastes the A4 sheets on the table using adhesive tape. The diagonals are marked and a circle is drawn using circular stencil having a hole in the centre coinciding with the centre on paper. The non-value added time is spent in extra processing for drawing a circle. Each trade carries their material bowls from one site to another and brings it back to the material station after work, causing transportation waste. Furthermore, the bowl's ingredients are placed in a disorganized manner, leading to extra motion of hands for searching the right block in the bowl. The use of defective blocks for roof work also leads to rework.

\section{Round 2}

Round 2 represents the use of Supermarket, 5S, Heijunka box and workplace design to improve the workflow and waste elimination. In this round, material is sorted initially by the quality manager, during when, defective pieces, small beads and shredded paper are removed. Only sorted blocks are kept on the material station in a well organised manner, which reduces excessive inventory, housekeeping, and rework issues. The batch size is reduced to 1 which means that the next trade can enter the site immediately after the previous trade finishes without much waiting. The material station adjoins the working 
station, which reduces unnecessary movement. Each Heijunka box is kept between two adjacent sites to fill the sorted Lego blocks as per the exact requirement only in the respective shelves for each site as well as trade. The quality manager fills the box every 50 seconds for every next two trades in 3 turns, thereby easing inventory management. The use of $5 \mathrm{~S}$ and co-location concepts reduces the time for searching the right blocks. The safety officer follows fewer steps to draw the circle by simply placing a different cardboard stencil (A4 size with circular cut-out of $50 \mathrm{~mm}$ radius). The glue stick replaces the use of adhesive tape to paste the A4 sheet. The overall workflow is improved but overproduction can still be observed.

\section{Round 3}

Round 3 shows the effects of pull planning, Kanban and workload balancing on the work flow. The rules in Round 3 pertaining to batch size and material sorting are similar to Round 2. In Round 3, the column casting is combined with foundation laying and the activity of putting roof studs is combined with blockwork laying. Thus, the player for column casting is removed from the game. Now, each player has similar cycle times as shown in Figure 5. Workload balancing reduces internal waiting and manpower use. In addition, all players now depend upon the last person completing their activity on the certain site to move to next site. Each player pastes a sticky note on their right after completing their activity on one site. Every player in the flow sees to their left side to check if the note (or Kanban) has been pasted to move to the next site. This means that no player can start work on the the next site until the work of the previous player has been completed. This reduces overproduction.

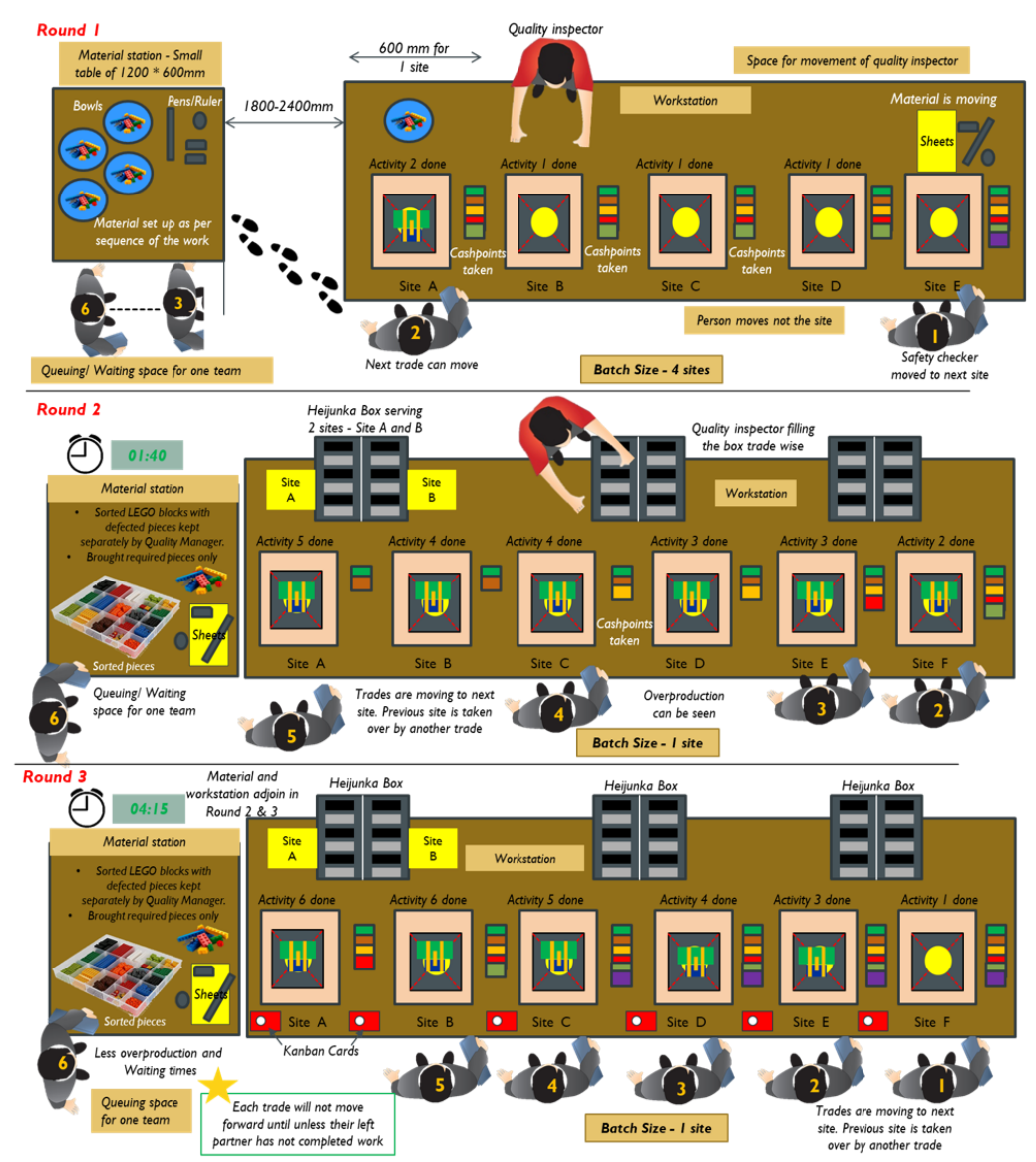

Figure 3: Sequence of work in different rounds 


\section{COSTING System}

The costing template is filled at the end of each round to calculate profit earned by each team on the basis of assumptions defined in this section. A total of 3000 cashpoints is given to each team's contactor head as advance for the construction of eight sites at the start of the game. The total cashpoints earned (profit) are calculated for the complete lead time. A positive sign (+) shows money given to or available with the contractor head while a negative sign (-) shows money given by the contractor head. The contractor head pays for the design cost, material cost, cost of each trade work/site, housekeeping, and rework. In return, he gets paid 400 cashpoints per site upon completion of each defectfree site irrespective of rework. The contractor head's profit is the difference between the expenditure and pay recieved. The team with higher profit or cashpoints wins the game.

\section{GAME TESTING}

The game was designed with the help of an internal testing group from the inception stage. The internal testing group consisted of 10-12 students of Master's program of various disciplines related to built environment. To test the prototype design and game sequence, numerous runs were played in small sections along the game development for analysing their behaviour towards the designed plot and to check their responsiveness to what is required or planned. The cycle times and scenarios were recorded with stopwatches and videography respectively to analyse how the game would proceed. At a later stage, a small part of game testing was performed with a different group due to pandemic constraints.

The testing was focussed on whether the game achieves the fulfilment of it's learning objectives namely waste elimination and analysis of Non-Value Adding (NVA) as well as Value-Adding (VA) activities. Time and cost were considered the drivers of the attention of the participants towards waste elimination and differentiation of non-value added activities, to streamline the workflow in the construction processes. The improvement in workflow by understanding of VA/NVA activities and waste reduction was expected to result in time and cost saving.

After playing Round 1, the players reported experiencing excessive waiting, unnecessary motion, extra-processing with added steps and inventory, overproduction before requirement and housekeeping issues. Round 1 had inconsistent flow due to the presence of lean wastes experienced by players. On the other hand, the use of supermarket and 5S concepts with Heijunka box and reduction of batch size in Round 2 streamlined the flow and increased workability by reducing the possible NVA activities in mainly safety checking, quality checking, roof work activities and material sorting. A few snapshots of testing are shown below in Figure 4.

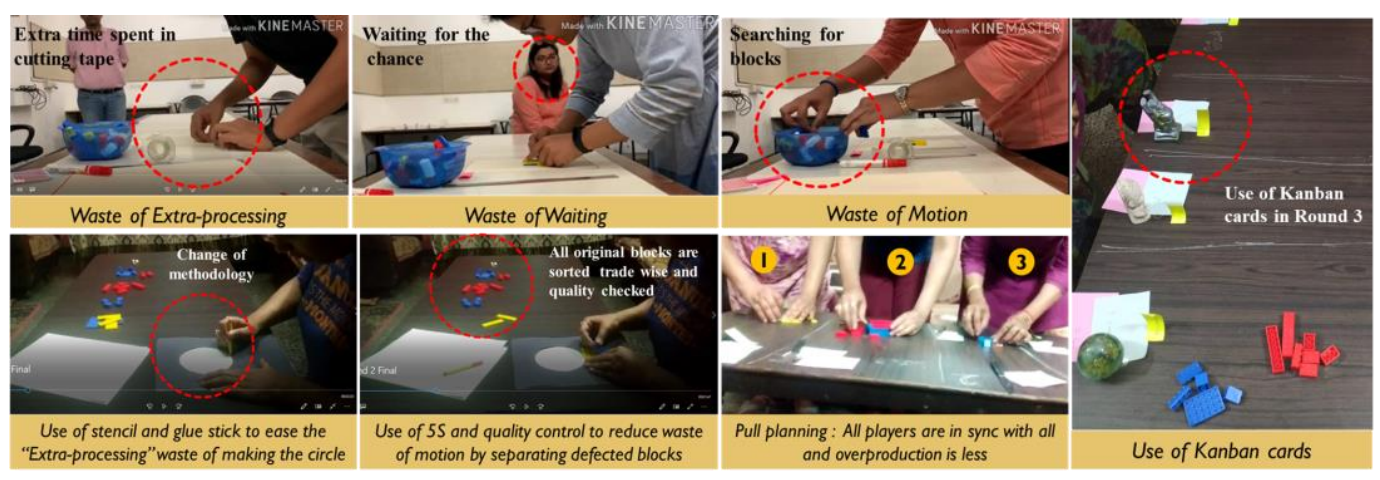

Figure 4: Snapshots from internal testing 
In Round 3, the introduction of pull planning and Kanban concepts balanced the overproduction and workload balancing brought further process optimisation and consistency in the workflow rather than showing huge cycle time reduction. This round showed how the similar tasks can be combined to bring cycle time for each trade close to each other to reduce waiting. The effect of improvement in workflow was analysed using the two variables - Time and Cost taken to complete the target. Round 1, 2 and 3 were played multiple times (approximately 4 - 5 runs) with the testing group and the average of the results for time and cost calculations were considered for analysis. The average cycle time of each trade to complete one site was recorded in the format card by timekeepers and is shown in the figure below.

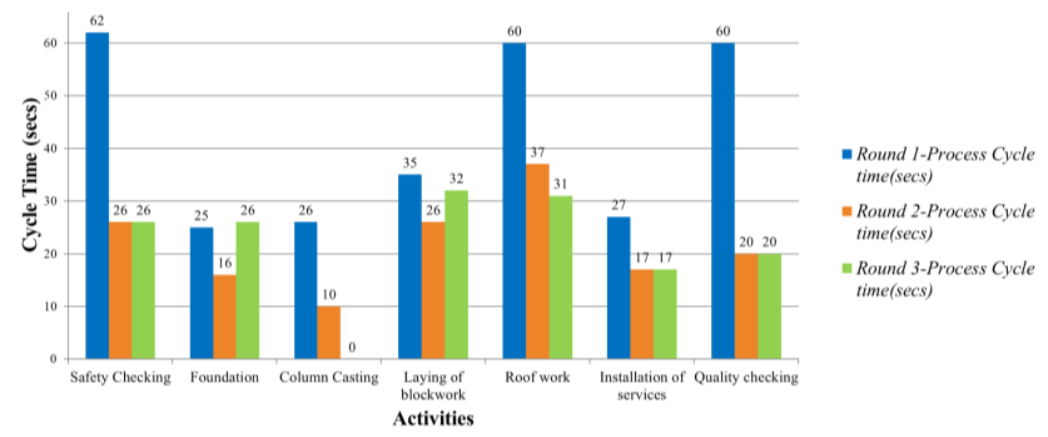

Figure 5: Cycle time comparison of each trade for each round

Considering the duration of game in mind, the game design adopted a simpler mechanism to map the process flow and lead time with the help of Gantt charts in an excel spreadsheet. After each round, the Gantt charts were prepared in the pre-formulated excel spreadsheet by timekeepers using average cycle time to determine the actual lead time taken by each team to complete the target of 8 houses. The spreadsheet had three worksheets for the three rounds. Each worksheet had the site number (A-H) in columns and the timeline (in seconds) in rows. Each cell was of square shape. Different colours were used to map the time taken by different players for each site. The Gantt chart showed the complete lead time and the process flow followed by each team during the game as shown in Figure 6. The resulting Gantt charts were projected on screen to the students and used as a point of discussion by the instructor for identifying VA/NVA activities, wastes, major bottle necks and problems, possible ways of cycle time reduction, and implementation process improvements as part of the debriefing sessions.

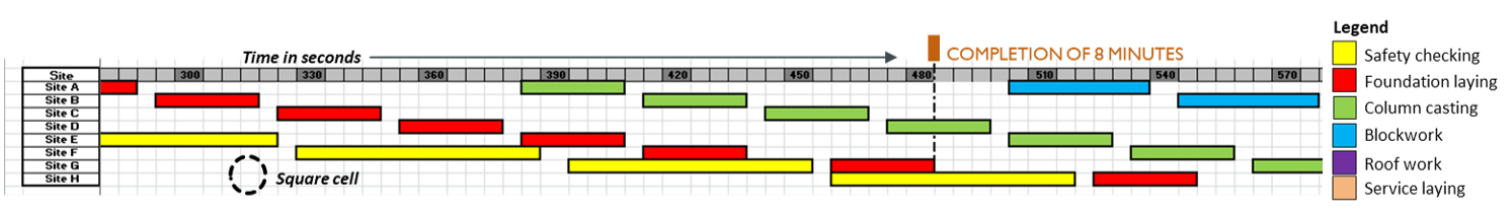

Figure 6: Part section of Gantt chart for Round 1

Cost templates were also filled by the contractor head of each team. The results of the Gantt chart and cost template in each round are summarised and analysed in the comparative statement shown in Table 1 . The Round 1 lasted 25 minutes while Round 2 and 3 were completed near the target time of 8 minutes. It has been observed that there were considerable improvements in Round 2 and 3 because the cycle time reduced by 35 to $67 \%$ for each trade. The improvements could be substantially attributed to the use of practices such as 5S, Supermarket, Heijunka box, and Kanban for waste reduction in Round 2 and Round 3. However, there is a possibility that improvement could also be, to 
a small extent, attributed to the increased familiarity of the participants with the game from Round 1 to Round 2 and 3. Cost is also another major driving force in construction industry and so was adopted in the game. As shown in comparative statement below, the profit increased over each round due to optimization of the quality as well as quantity of materials. This led to a manageable inventory and fewer reworks.

Table 1: Comparative statement of results of each round

\begin{tabular}{lccc}
\hline \multicolumn{1}{c}{ Parameters } & Round 1 & Round 2 & Round 3 \\
\hline Profit earned (cashpoints) & 2710 & 3700 & 3940 \\
Time taken to complete task (min.) & 25 & 8.2 & 7.4 \\
Cashpoints earned per minute & 108 & 453 & 531 \\
Cashpoints made in 8 minutes & 864 & 3624 & 4248 \\
\hline
\end{tabular}

The flow simplification impacted the time as well as the cost. The lead time over the three rounds reduced considerably from 25 minutes to 7.4 minutes and accounted to more than $300 \%$ improvement. The rate at which the profit was being earned over the rounds increased by $500 \%$ i.e. $(4248 / 864=4.92)$. This means that the same amount of work can be performed in one third of the time and five times more money can be gained as compared to first round. So, the profitability was high in the later rounds. Game testing showed that maximising time spent on value-adding activities such as building the block and minimising time spent on non-value added activities like moving things around, searching for the right block, waiting and quality checking in the later rounds reduces the cycle times of each trade, thus, leads to time as well as cost saving.

\section{POST SIMULATION DISCUSSION}

At the end of each round, a 15-minute debriefing session was conducted by the instructor with help of the assistants and timekeepers. The interactions were in the form of group discussion and collecting "plus-delta" to summarise their learnings and share their experiences of the game. For consistency, the instructor used a 12 pointer debriefing questionnaire that helps in systematic perspective analysis of the participant's learning outcomes. The questionnaire focussed on highlighting VA/NVA activities and waste elimination concepts, real life applications of these concept and receiving feedback on the game design in terms of simplicity, ease, fun element, etc. The discussion on process flow with respect to waste and VA/NVA activities were assisted by the Gantt charts shown on projector screen. The impact of actions was linked to the time and cost variables. A collective feedback was collected from each team first and later shifted to the specific players regarding their opinion and experience. The plus points of the feedback were regarding the design of material bowls used in Round 1, use of house prototype and cost variable for the game. The participants reiterated the seamless and gradual infiltration of the concepts in the game; the type of wastes was understood in Round 1; the usefulness of 5S, supermarket and Heijunka box was understood in Round 2; the efficacy of Kanban and Pull planning was seen in Round 3. The deltas were regarding the space arrangement and use of different colours for different trades in a single design code. Later, a single colour was decided for all blocks for a design code to avoid complicated flow. Based on the richness of the discussion, it was evident that the simulation was effective in providing a first-hand experience of waste elimination and value maximisation by the use various lean practices. 


\section{CONCLUSION}

This game was developed with the intent to impart knowledge on lean philosophy concepts such as waste elimination and value maximisation. Testing of the game showed that participants understood these concepts after playing it. The efficacy of application of these concepts on important variables such as time and cost overrun was also understood by the participants. This game could help in bringing clarity to the minds of the participants to proceed confidently in future systematic identification and elimination of waste in real life construction processes. The preparation of Gantt charts provided participants with the opportunity to learn graphical representation of process mapping and will help them switch to more advanced ways of mapping for real life scenarios. The limitation of this study is that it used a simplistic mapping mechanism of time and flow with the help of Gantt charts. The knowledge gained from this game can be enhanced further in the form of current and future state maps by integrating the aspect of value stream mapping in a simulated form.

\section{REFERENCES}

Alarcón, L. F. (1995). Training Field Personnel to Identify Waste and Improvement Opportunities in Construction. 3rd. Annual Conference International Group for Lean Construction, University of New Mexico, Albuquerque, USA, 16-19 October.

American Society of Civil Engineers. (2021). Research Papers. ASCE Journals, Browse Journals by Titles and by Topics $\mid$ ASCE Library.

Bhatnagar, S. (2020). Lean Simulation Game - Exploring a perspective to study Value Stream Mapping. Graduate Thesis, CEPT University, Ahmedabad, India.

Demirkesen, S., Wachter, N., Oprach, S., \& Haghsheno, S. (2019). Identifying Barriers in Lean Implementation in the Construction Industry. 27th Ann. Conf. of the Intl. Group for Lean Construction (IGLC). Dublin, Ireland, 3-5 Jul 2019, 157-168.

Hamzeh, F., Theokaris, C., Rouhana, C., \& Abbas, Y. (2017). Application of hands-on simulation games to improve classroom experience. European Journal of Engineering Education, 42(5), 471-481. doi.org/10.1080/03043797.2016.1190688.

International Group for Lean Construction. (2021). Conference Papers. IGLC.net - Index.

Koskela, L. (2000). An exploration towards a production theory and its application to construction. VTT Publications.

Lean Construction Institute. (2021). LCI Simulation Matrix. LCI-Simulation-Matrix.pdf (leanconstruction.org).

Liker, J. K. (2004). The Toyota Way: 14 Managment principle from the world's greatest Manufacturer. In McGraw-Hill, New York.

Pollesch, P., \& Rovinsky, A. (2017). House of Cards - A Simulation of Lean Construction Principles. 25th Annual Conference of the International Group for Lean Construction. Heraklion, Greece, 9-12 Jul 2017, 373-380.

Rybkowski, Z. K., Forbes, L. H., \& Tsao, C. C. Y. (2018). The Evolution of Lean Construction Education (Part 1 of 2): At US-based Universities. 26th Annual Conference of the International Group for Lean Construction. Chennai, India, 18-20 Jul 2018, 1013-1023. doi.org/10.24928/2018/0447.

Visionary Products USA, Inc. (2021). Lean Zone® Production Methodologies. Lean Zone ${ }^{\circledR}$ Production Methodologies (lean-zone.com). 